\title{
The Metal-Poor IMF, Stellar Evolution, and Star Formation Histories
}

\author{
Evan D. Skillman ${ }^{1}$ \\ ${ }^{1}$ Astronomy Department, University of Minnesota, 116 Church St. SE, Minneapolis, MN 55455 \\ email: skillman@astro.umn.edu
}

\begin{abstract}
I present an introduction to three important subjects relevant to low metallicity star formation: the IMF, stellar evolution, and star formation histories. I will draw on observations from the LCID (Local Cosmology from Isolated Dwarfs) project to illustrate some of these topics.
\end{abstract}

Keywords. stars: evolution, galaxies: evolution, galaxies: dwarf, (galaxies:) Local Group

\section{Introduction}

I was asked by the organizing committee to give an introductory lecture to the "IMF, Stellar Evolution, and Star Formation Histories," section of the conference program. Obviously a comprehensive overview of these three important topics is not possible here. Instead I settled on a few comments which I hoped might provoke some thought. I entertained myself by including references to work by all of the section's speakers in the talk, but some of those may have not been propagated through to this write-up.

\section{The Initial Mass Function (IMF)}

"Testing the Universality of the IMF remains as our primary challenge for the coming decade" Kennicutt (1998).

This quote comes from a review by Kennicutt, which provided the lead-off talk to a conference dedicated to the study of the IMF. The bulk of that talk focused on the importance of the IMF to just about every possible problem in astrophysics. Although ten years old, I highly recommend the talk as a good starting point for understanding the importance of the IMF and the biggest challenges in its study.

\subsection{Do we expect the IMF to change with metallicity?}

It is likely that the IMF is a reflection of the ISM from which the stars are born. Thus, characterizing the properties of the ISM at low-metallicity is vital to our understanding of the star formation process, and, accordingly, galaxy formation and evolution.

The chemical composition and content of the ISM in low-metallicity systems are fundamentally different than those of massive metal-rich galaxies. The dust and molecular gas content of galaxies are commonly assumed to scale with metallicity, with the most metalpoor systems being essentially devoid of dust, and showing a relative under-abundance of molecular gas. In massive galaxies, dust and molecular gas play a crucial role in the formation of stars, and yet, many low mass systems are actively forming stars in the assumed relative absence of dust and molecular gas.

Theoretical arguments indicate that the nature of the ISM in galaxies changes as a function of metallicity. For example, Norman \& Spaans (1997) and Spaans \& Norman (1997) emphasize a "phase transition" which occurs in the range of $\mathrm{Z} \approx 0.03-0.1 \mathrm{Z}_{\odot}$. In 
their models, the ISM at lower metallicities is characterized by a single temperature, and the familiar multiphase medium with stable cool and warm components does not appear until the metallicity has sufficiently increased. Thus, at lower metallicities, star formation is suppressed with obvious implications for the evolution of dwarf galaxies. There are also several theoretical studies which show that the character of photodissociation regions changes significantly as a function of metallicity, e.g., van Dishoeck \& Black (1988), Maloney \& Black (1988), Lequeux et al. (1994), Bolatto et al. (1999), Röllig et al. (2006).

Observational evidence is accumulating which supports the impression that the character of the ISM is different at lower metallicities. In the more than two decades since the earliest observations in dwarf galaxies by Elmegreen et al. (1980) and Tacconi \& Young (1987), CO has not been detected in any galactic environment with $12+\log (\mathrm{O} / \mathrm{H})$ $<\sim 8.0$. Taylor et al. (1998) have claimed that there is a strong transition below Z $\approx 0.1 \mathrm{Z}_{\odot}$, where $\mathrm{CO}$ emission drops dramatically relative to galaxy properties such as size and luminosity. The lack of CO detections at lower metallicities was recently confirmed in a large survey for CO in dwarf galaxies by Leroy et al. (2005) and a very deep observation of the extremely metal-poor galaxy I Zw $18(12+\log (\mathrm{O} / \mathrm{H})=7.17$, Skillman \& Kennicutt (1993)) by Leroy et al. (2007). There is on-going debate about the metallicity dependence of the conversion from $\mathrm{CO}$ emission to $\mathrm{H}_{2}$ mass, see e.g., Cohen et al. (1988), Elmegreen (1989), Rubio et al. (1993), Wilson (1995), Verter \& Hodge (1995), Arimoto et al. (1996), Bolatto et al. (2003), Leroy et al. (2006), Bolatto et al. (2008), and references therein, with the result that the $\mathrm{H}_{2}$ masses of low metallicity dwarf galaxies are still not well known. Nonetheless, the complete absence of CO emission in dwarf galaxies showing a strong presence of star formation indicates that something is different at these lower metallicities.

Another indication that the nature of the ISM changes at low metallicity comes from the recent surveys measuring the diffuse $8 \mu \mathrm{m}$ emission from hot $(\sim 350 \mathrm{~K})$ dust and PAHs, i.e., Engelbracht et al. (2005), Hogg et al. (2005), Jackson et al. (2006), Rosenberg et al. (2006). Engelbracht et al. (2005) showed that an abrupt change in the properties of the ISM occurs at a metallicity $1 / 3$ to $1 / 5$ of the solar value. They showed that the ratio of the 8 to $24 \mu \mathrm{m}$ luminosities dropped significantly in galaxies below this metallicity, which was attributed to the weakening of the PAH features at low-metallicity. Jackson et al. (2006) showed that this transition metallicity coincides with a drop off of other ISM components, namely the molecular gas content, and also that the slope of the diffuse $8 \mu \mathrm{m}$ luminosities below this metallicity continues to very low metallicity (over one dex below the Engelbracht et al. (2005) transition metallicity).

Although the paucity of diffuse $8 \mu \mathrm{m}$ emission at low-metallicity is well established, the Spitzer IRAC $8 \mu \mathrm{m}$ imaging programs that have been so efficient at detecting this emission have had the major drawback that they do not distinguish between emission from the $7.7 \mu \mathrm{m}$ PAH feature and the continuum emission from hot dust grains. Cannon et al. (2006) showed that one region in the dwarf starburst galaxy NGC 1705 that emitted strongly at $8 \mu \mathrm{m}$ was dominated by emission from PAHs, while another region emitted only hot dust continuum radiation with no detected PAH emission. The absence of PAHs can be a result of the hard radiation fields that are pervasive in low-metallicity starburst systems destroying PAHs, see Madden et al. (2006), although it is not clear why in some cases the PAHs survive, e.g., Cannon et al. (2006). What is even less well understood are the relative contributions of $\mathrm{PAH}$ and hot-dust continuum emission in typical quiescent dwarf galaxies, which have very different radiation fields than starburst galaxies.

PAH emission is typically observed along the outer edges of PDRs, e.g., Giard et al. (1994), Helou et al. (2004), where they are thought to be transiently heated by single highenergy photons, c.f., Leger \& Puget (1984). However, Lemke et al. (1998) observed PAH 
emission in a region of the Galaxy illuminated by only the typical interstellar radiation field of the solar neighborhood. Thus, it is clear that strong radiation fields do excite PAHs into emission, but it is not yet clear if they are required to excite them. Smith et al. (2007) recently published a comprehensive spectroscopic study of PAH emission in 59 galaxies over an impressive range of host galaxy metallicities and galaxy morphological types. They found a dependence of the relative PAH band strengths on host galaxy metallicity with the longer wavelength $\mathrm{PAH}$ bands being relatively weaker at lower metallicity, which was attributed to the inability of large PAHs to survive in low-metallicity environments.

Finally, the abundance of dust has long been known to depend strongly on the metallicity of the environment. Although the abundance of dust becomes low at low metallicities, recent observations have shown some evidence for dust even in the lowest metallicity environments known. Cannon et al. (2002) find $\sim 10^{3} \mathrm{M}_{\odot}$ of dust in the most metal-deficient galaxy known, I Zw 18. A similar abundance of dust is found in the second most metalpoor galaxy SBS 0335-052, see Dale et al. (2001), Houck et al. (2004). While the results are encouraging, it is important to note that almost all of the available information on the properties of dust at very low metallicities comes from either reddening or UV absorption observations. A comparatively unexplored area of the low-metallicity ISM is the far-IR emission from dust. This spectral region radiates the bulk of the bolometric luminosity from normal galaxies, e.g., Calzetti et al. (2000), and allows one to trace the locations of dust with much better precision than is available with other techniques. Previous far-IR investigations have typically excluded dwarf systems due to their lower surface brightness compared to more massive galaxies. Nonetheless, the majority of Local Group dIrr galaxies were detected by IRAS, c.f., Melisse \& Israel (1994), Lisenfeld \& Ferrara (1998).

In Marco Spaans' talk, he showed models where a phase transition occurred at metallicities between $1 \%$ and $0.1 \%$ of the solar value. Most of the above evidence indicates that major changes happen in the ISM at metallicities which are closer to $10 \%$ of the solar value. During later discussions at the conference, Marco indicated that the transition metallicity is a strong function of the ISM porosity, so a more porous ISM will experience the phase transition at higher metallicities (than the models that he showed). Thus, it may be possible that the observed changes could be interpreted within his phase transition model.

\subsection{Is there evidence for a metallicity dependence of the upper IMF cut-off?}

At metallicities of roughly the solar value, several studies suggest an upper limit to the IMF of $\approx 120-200 \mathrm{M}_{\odot}$, e.g., Weidner \& Kroupa (2004), Oey \& Clarke (2005), Figer (2005), but see also Melena et al. (2008). Despite a rather convincing figure in Figer (2005), this result is not without controversy, but it does provide a good start for this discussion.

The reason that there is controversy, even within our own galaxy, is that there are several criteria that an observation of a cluster must meet in order to be decisive on this point. First, the cluster must be young enough that the most massive stars have not yet evolved away. Second, the cluster must be massive enough that the upper IMF is completely populated. Third, the spread in the formation ages of the stars must be less than the lifetimes of the most massive stars. It is not clear that there are any known clusters which fulfill these three criteria without question. Additionally, luminosities and spectra must be converted into indicative masses with confidence. Some would argue that spectra are required for every star in order to meet this criterion, making this type of study very expensive in terms of telescope time, even in our own galaxy.

Given the above, is there any hope of making similar measurements at significantly lower metallicities? Specifically, can we make this type of observation at metallicities 
below where we might expect a phase transition in the ISM? In fact, this type of observation in an environment with a metallicity less than $10 \%$ of the solar value becomes considerably more difficult solely due to distance. The nearest low-metallicity, star forming environments (below one-tenth solar, where one might expect a putative phase change in the ISM) - the isolated dwarf irregular galaxies of the Local Group, e.g., Mateo (1998) are ten times further away than the Magellanic Clouds.

Are there other ways to probe the upper IMF beside a strict stellar census in a cluster? From $\alpha /$ Fe ratios in stars, Tolstoy et al. (2003) suggest that they are seeing evidence for a truncated IMF in dSphs. However, Koch et al. (2008) point to the important roles of metal-enhanced winds and inhomogeneous mixing in the interpretation of $\alpha / \mathrm{Fe}$ ratios, and question the uniqueness of the Tolstoy et al. hypothesis in interpreting the observations. As more observations of precision relative abundances become available in the dSphs, it may be possible to identify trends in the plots of $\mathrm{X} / \mathrm{Y}$ vs. Fe/H to constrain the possible physical processes which give rise to these trends. From the small sample that Tolstoy et al. (2003) had to work with, the suggestion of a truncated IMF must be regarded as speculative, but the quantity of the data available is increasing rapidly.

There is really quite a bit at stake here. For example, Garnett (2002) has demonstrated that the effective oxygen yields of star forming dwarf galaxies increase with the mass of the galaxy. This is usually interpreted as due to the effect of metal enhanced winds flowing out of the small gravitational potential wells of the smallest galaxies. However, a metallicity dependent upper IMF truncation would have an identical effect.

\subsection{Is the upper IMF slope metallicity dependent?}

Leaving the upper mass cut-off, is it possible to constrain the slope of the upper IMF? Historically, the answer has been that there is no firm evidence for departures from the "universal" IMF, but this is also observationally very difficult. Naively, one would think that one can measure very large numbers of stars in nearby galaxies, and thus, be able to constrain the IMF. In practice, this becomes very difficult because of the degeneracy between the star formation history and the IMF. This is discussed in great detail in Miller \& Scalo (1979), and, more recently, in Elmegreen \& Scalo (2006).

In Fig. 1, from a study of NGC 5128 by Rejkuba et al. (2004), one can see that for color-magnitude diagrams of photometric depths typical of nearby galaxy studies that the constraints on the IMF are relatively weak. In the figure it is clear that reasonable fits to the main sequence luminosity function are possible over a large range in IMF slope.

If a large range of IMFs are consistent with the data typically observed in nearby galaxies, does that weaken the conclusions one derives from the study of the color-magnitude diagrams? The silver lining here is that the reconstructed star formation rate is not strongly dependent on the assumption of the IMF. Figure 6 of Angeretti et al. (2005) shows an example where the calculated star formation rate of NGC 1569 changes by less than a factor of two over a range in the IMF slope from $\sim 1$ to $\sim 2.5$. Of course, when the assumed IMF exceeds $\sim 2.7$, the star formation begins to shoot up dramatically. Nonetheless, it is comforting that a reasonably large range in the assumed IMF results in rather stable calculated star formation rates.

\section{Stellar Evolution}

"Massive stars played a key role in the early evolution of the Universe... It is therefore very important to understand their evolution." Hirschi et al. (2008)

"We hope to encourage observers to provide stronger observational constraints where they are needed .." Gallart et al. (2005) 


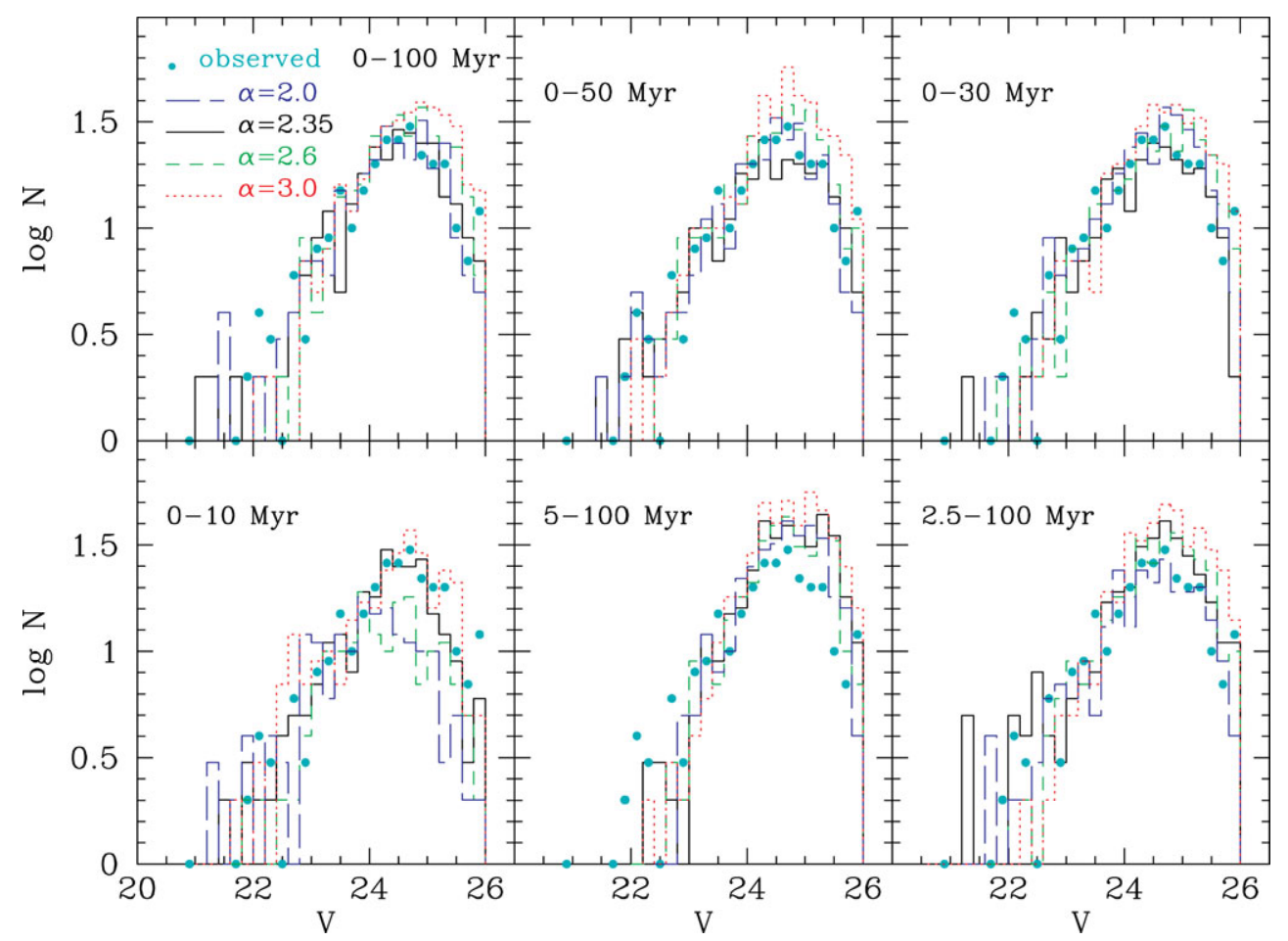

Figure 1. The observed Main Sequence (MS) luminosity function (dots) is compared with synthetic luminosity functions for different SFHs for $Z=0.008$ and four different IMF slopes $(\alpha=2.0,2.35,2.6$, and 3.0) as indicated in the legend. Synthetic luminosity functions correspond to models calculated adopting the appropriate distance, reddening and a constant SFR in the interval that is indicated in each panel (beginning-end of the burst). In the observed and simulated luminosity functions color and magnitude cuts are applied, and only stars bluer than $U-V=-0.7$ and brighter than $V=26$ are compared (figure and caption from Rejkuba et al. 2004).

The first quote prompted my observation that the astronomical community is still dangerously low in the resources allocated to research on stellar evolution. Practically everything that we study is dependent to some degree on the results of stellar evolution models. As this modelling continues to explore new physical parameters (e.g., rotation, winds) the hope is that the models approach better representations of nature. We heard of great progress from new models in the talks by Leitherer and Ekström.

In this section I review some of my own experience with using the results of stellar evolution models, and close with what I believe is a promise to follow-on to the encouragement given in the second quote.

\subsection{Using HST to map recent star formation in dwarf galaxies: testing our knowledge of the star formation process}

My closest connection to stellar evolution research is my use of stellar evolution models to recreate recent star formation histories of nearby dwarf galaxies. Here I review the power of combining Hubble Space Telescope (HST) photometry with models of stellar evolution to produce spatially resolved recent star formation histories of galaxies with time resolution of order $30 \mathrm{Myr}$ over the last few hundred Myr. 
The first HST color magnitude diagram of the stars in the nearby dwarf irregular galaxy Sextans A by Dohm-Palmer et al. (1997) revealed for the first time a clear separation between the brightest main sequence stars and the blue helium burning (BHeB) stars intermediate and high mass stars that have evolved beyond the main sequence, ignited helium burning in their cores, and have migrated back to the blue side of the color magnitude diagram. The separation of the main sequence stars and the $\mathrm{BHeB}$ was made possible by the high angular resolution of the HST (reducing photometric errors due to blends) and the low metallicity of Sextans A which leads to low differential reddening.

These BHeB stars afford a special opportunity to study the recent star formation histories of nearby galaxies. Because the position of a BHeB star in the CMD represents a unique age (as opposed to, for example, the main sequence or the red giant branch where a single position can correspond to a large range of ages), one can convert the $\mathrm{BHeB}$ luminosity function directly into a star formation history (SFH) (with the assumption of a universal initial mass function). The main limiting factor of this technique is that the position of the $\mathrm{BHeB}$ stars blends into the red clump at an age between 0.5 and 1 Gyr. Because one knows the positions of the BHeB stars in the galaxy, then one can produce a spatially resolved SFH (i.e., it is possible to produce "movies" of the recent star formation).

Translating the BHeB star luminosity function into a SFH depends on the accuracy of the stellar evolution models. We have different lines of evidence that the stellar evolution models provide an excellent guide to this stage of evolution. The first line came from the early HST observations themselves. Although the position of the blueward extension of the stellar evolution tracks is a strong function of both metallicity and stellar mass, excellent agreement between observations and models was found by choosing the stellar evolution tracks for the metallicity determined from the HII region abundances for Sextans A. This is a very important point. There are no low metallicity, young stellar clusters in the Milky Way galaxy or the Magellanic Clouds which allow the stellar evolution modelers to calibrate their codes in this regime (the oxygen abundance in Sextans $\mathrm{A}$ is a factor 3 lower than in the Small Magellanic Cloud). When these stars are first observed in the extragalactic context, the agreement with models can be taken as confirmation of a prediction.

Dohm-Palmer et al. (2002) used deeper HST photometry of Sextans A to compare recent star formation histories recovered from both the main-sequence stars and the $\mathrm{BHeB}$ stars for the last $300 \mathrm{Myr}$. The excellent agreement between these independent star formation rate (SFR) calculations is a resounding confirmation for the legitimacy of using the BHeB stars to calculate the recent SFR. Dolphin et al. (2003) derived the recent SFH of Sextans A from the entire CMD and found good agreement with that derived from the BHeB stars alone.

Additionally, Dohm-Palmer \& Skillman (2002) used all of the HST observations of Sextans A in order to compare the ratio of blue to red supergiants. This ratio provides an observational constraint on the relative lifetimes of these two phases that is a sensitive test for convection, mass-loss, and rotation parameters. Analyzing the ratio as a function of age, or, equivalently, mass eliminates the confusion of unknown star formation histories (as in previous studies of this ratio). The functional form of the observed ratio matches the model extremely well with an offset of roughly a factor of 2 (and the offset is seen as support of the latest models which include rotation). Given these tests of reliability of the stellar models, we feel confident that using the BHeB stars to construct recent star formation histories is well justified.

Dohm-Palmer et al. (1998) employed HST photometry of four nearby dwarf irregular galaxies (Sextans A, Leo A, Pegasus, and GR 8) and derived recent star formation 


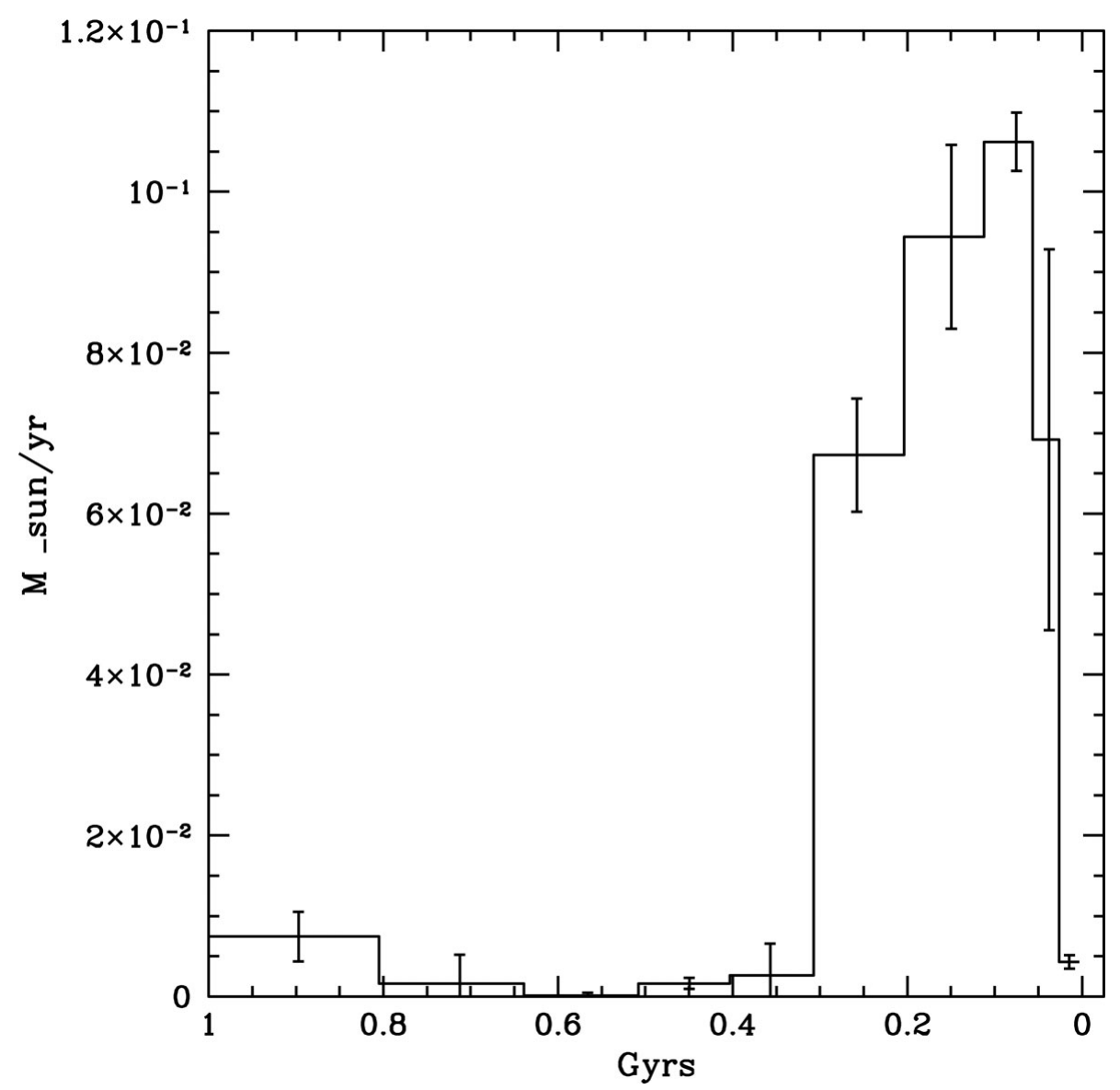

Figure 2. The recent SFH for the M81 dwarf galaxy DDO 165. Here we see an intense burst of star formation that ended just recently. The burst lasted for roughly $200 \mathrm{Myr}$.

histories for these galaxies. At the time, the surprising result was the lack of bursts or episodes of enhanced star formation. With time bins of only $25 \mathrm{Myr}$ for the last 500 Myr, with the possible exception of enhanced star formation rates in the last $50 \mathrm{Myr}$ for Sextans A and Leo A, all four galaxies are best described as nearly constant star formation rates. In retrospect, perhaps the lack of truly zero star formation rates is as surprising as the lack of periods of enhanced star formation.

\subsection{HST ACS observations of M81 Group dwarfs}

My collaborators and I have been able to continue this work through an HST program to study the star forming dwarf galaxies of the M81 Group. Studies of the impact of star formation ('feedback') on the properties of a galaxy are of fundamental importance to understanding galaxy evolution. One crucial aspect in these studies is a precise census of the recent star formation in a galaxy. We have recently obtained HST ACS observations of a sample of M81 Group dwarfs which allow deriving spatially resolved star formation histories with a time resolution of roughly $30 \mathrm{Myr}$ over the last $500 \mathrm{Myr}$, see Weisz et al. (2008). Our sample comprises nine galaxies in the M81 group which is host to a wide diversity of dwarf star forming galaxies. They span ranges of 6 magnitudes in luminosity, 1000 in current star formation rate, and 0.5 dex in metallicity. The ACS 
observations allow us to directly observe the strength and spatial relationships of all of the star formation in these galaxies in the last $500 \mathrm{Myr}$. We can then quantify the star formation and measure (1) the fraction of star formation that is triggered by feedback, (2) the fraction of star formation that occurs in clusters and associations, and (3) to what degree star formation is governed by the feedback from star formation. The ACS observations will be complemented with high-quality ancillary data collected by our team for all galaxies (e.g., Spitzer, UV/optical/NIR, VLA HI). This will enable us to construct prescriptions of how star formation and feedback depend on metallicity, size, gas content, and current star formation rates in galaxies. As an example of the recent star formation history reconstructions, Fig. 2 shows the recent SFH of DDO 165, revealing a rather dramatic burst of star formation in the recent past.

In working on this new sample of galaxies, one of the things that we have discovered is that the stellar models do not follow the tracks of the BHeB stars nearly as well as in our studies of the low metallicity Local Group galaxies. To first order, the tracks predict the positions well; the trends predicted by changing metallicities are seen, but the detailed shapes in the color magnitude diagrams show some discrepancies. In the spirit of the Gallart et al. quotation, we will provide the stellar modellers with new constraints on this phase of stellar evolution. Finally, I note that the HST ACS Nearby Galaxy Survey (ANGST, PI Dalcanton) has provided new, high quality observations of a larger sample of nearby galaxies, so these constraints will grow in quantity.

\section{Star Formation Histories}

"The lack of a comprehensive scenario for the formation and evolution of dwarf spheroidal galaxies contrasts with the large amount of available data for these nearby Local Group satellites," Salvadori et al. (2008), and see also de Rijcke et al. (2005)

My work on star formation histories of galaxies has been almost exclusively confined to dwarf galaxies, which I will review here. Although the quote above sets a rather harsh view, I believe that significant progress has been made in the last decade.

\subsection{Lifetime star formation histories of dwarf galaxies}

The nearby dwarf galaxies of the Local Group are a unique probe of the chemical and structural evolution of the universe over its entire history. Interpretation of the fossil evidence contained within these systems provides direct and quantitative constraints on their star formation histories (SFHs) and broader evolution (e.g., Mateo 1998). However, dwarf galaxy evolution is dependent upon both local and cosmic environmental factors. It is therefore of tremendous importance to disentangle these effects for the nearby galaxies so that key results obtained locally can be correctly interpreted in a wider cosmological context. For example, it is important to understand whether the SFH of a dwarf galaxy is affected more by the radiation field of a nearby massive galaxy or by the diffuse background radiation field from the epoch of cosmic reionization.

The position - morphology relation seen for the lowest-mass systems in the Local Group indicates that local environmental factors can be as important as the generic cosmic structure formation framework; gas-poor, pressure-supported dwarf galaxies (dwarf spheroidal, dSph) are preferentially found as satellites to the MW and M31, whereas gas-rich rotating systems (dwarf irregular, dIrr) are preferentially found in isolated locales, e.g., van den Bergh (1994a). Additionally, the closest MW dSph companions, like Draco and Ursa Minor have only old stellar populations with ages $\geqslant 10$ Gyr, whereas those at a distance of $100 \mathrm{kpc}$ or greater, like Fornax or Leo I have prominent young and intermediate-age stellar populations see, e.g., van den Bergh (1994b). 
Detailed dynamical modeling of the orbital evolution of dwarf satellites provides clues to the role of local environment in dwarf galaxy evolution, c.f., Mayer et al. (2001a), Mayer et al. (2001b), Mayer et al. (2006). "Tidal stirring," which is a combination of tidal effects and disk shocking, can remove most of the gas from a dwarf galaxy and transform rotationally supported systems into pressure supported systems. Essentially, this will convert a dIrr galaxy into a dSph galaxy. The tidal stripping of both baryonic and dark matter from satellites is also known to occur, and while this process alone is unlikely to change the morphology of a system from a dIrr to a dSph, it will still change the observable properties of the dwarf, as spectacularly demonstrated by the Sagittarius dwarf galaxy. Finally, there is some evidence for a link between tidal interactions and star formation, such that tides are able to trigger star formation. All of these effects suggest that the observed properties of dwarf galaxies should be a complex function of their spatial location and orbital properties within the group where they are observed.

I am part of a cycle 14 HST program (LCID or Local Cosmology from Isolated Dwarfs) in which five isolated, Local Group dwarfs were observed to depths below the oldest main sequence turnoffs. Matteo Monelli's contribution to these proceedings gives a nice overview of the project and its goals. In this program, Cole et al. (2007) have uncovered one dwarf (Leo A) with evidence of suppression of star formation at early ages - resembling the expected signature of cosmic reionization. The dwarf irregular galaxy also shows evidence of delayed star formation, as seen in an earlier WFPC2 study by Skillman et al. (2003), although the new ACS observations allow for better time resolution and smaller errors on the star formation rates. The isolated dSph galaxies, Cetus and Tucana, show almost exclusively old stars attributed to an episode of star formation in the first few billion years of the age of the universe. The one complication with their color magnitude diagrams is the presence of a blue straggler population. It is not clear whether these can be interpreted as very low level star formation at later ages, or the result of primordial binary stars as discussed in Momany et al. (2007) and Mapelli et al. (2007). Finally, the "transition" galaxies, LGS-3 and Phoenix, are intermediate between with regard to star formation histories. They both show predominantly early star formation, but continue to form stars at a significantly level up to the present.

\subsection{IMF constraints from LCID observations}

Because of the exceptional quality of the LCID photometry, I was curious to see what constraints could be gained on the IMFs of the observed dwarfs. Sebastian Hidalgo ran a number of experiments, fitting the detailed color magnitude diagrams with different values of the IMF slope and then determining the $\chi^{2}$ for the best possible fits. We concentrated these tests on LGS-3 and IC 1613. Because of the low level of recent star formation in LGS-3, the IMF slope could be changed over a large range (from zero to 5) with only very small changes in the $\chi^{2}(1.05$ to 1.2$)$. This clearly showed that the best constraints on the IMF will come from galaxies with strong star formation at all ages. For IC 1613 we found that the $\chi^{2}$ was much more sensitive to the IMF, bounding the slope to values between one and three. Given the quality of the photometry, this loose constraint may come as a disappointment. On the positive side, for the range of IMF values which gave satisfactory $\chi^{2}$ values, the star formation histories appear nearly identical. There is a small degree of transferring early star formation for late star formation (as expected), but the overall trend with time remains preserved. 


\section{Summary}

I'm not sure that a summary is appropriate for a introductory talk which is really just a collection of thoughts. However, at the conference I did emphasize that the Local Group and nearby dwarf galaxies can provide us with star formation histories for a significant number of galaxies sampling a range of environments. The range of metallicities in these nearby galaxies is large. In fact, some of them (e.g., Leo A and Sag DIG) are comparable to the low metallicity of I Zw 18, see, Skillman et al. (1989), Saviane et al. (2002), van Zee et al. (2006). This large range in metallicity in nearby galaxies allows us to search for metallicity dependences in the star formation process. The lifetime star formation histories that are available from the HST allow us to identify the physical processes that determine a galaxy's current evolutionary state (which we refer to as morphology). Interestingly, we now have very clear evidence that the small dwarf irregular galaxies have had significant delays in their star formation relative to the dSphs.

\section{Acknowledgements}

Finally, I would like to thank the conference organizers for a fantastic experience and my many collaborators for their efforts.

\section{References}

Angeretti, L., Tosi, M., Greggio, L., Sabbi, E., Aloisi, A., \& Leitherer, C. 2005, AJ, 129, 2203

Arimoto, N., Sofue, Y., \& Tsujimoto, T. 1996, PASJ, 48, 275

Bolatto, A. D., Jackson, J. M., \& Ingalls, J. G. 1999, ApJ, 513, 275

Bolatto, A. D., Leroy, A., Israel, F. P., \& Jackson, J. M. 2003, ApJ, 595, 167

Bolatto, A. D., Leroy, A. K., Rosolowsky, E., Walter, F., \& Blitz, L. 2008, ArXiv e-prints, 807, arXiv:0807.0009

Calzetti, D., Armus, L., Bohlin, R. C., Kinney, A. L., Koorneef, J., \& Storchi-Bergmann, T. 2000, ApJ, 533, 682

Cannon, J. M., Skillman, E. D., Garnett, D. R., \& Dufour, R. J. 2002, ApJ, 565, 931

Cannon J. M., et al. 2006, ApJ, 647, 293

Cohen, R. S., Dame, T. M., Garay, G., Montani, J., Rubio, M., \& Thaddeus, P. 1988, ApJL, 331, L95

Cole, A. A., et al. 2007, ApJL, 659, L17

Dale, D. A., Helou, G., Neugebauer, G., Soifer, B. T., Frayer, D. T., \& Condon, J. J. 2001, AJ, 122,1736

de Rijcke, S., Michielsen, D., Dejonghe, H., Zeilinger, W. W., \& Hau, G. K. T. 2005, A\&A, 438, 491

Dohm-Palmer, R. C. \& Skillman, E. D. 2002 AJ 123, 1433

Dohm-Palmer, R. C., Skillman, E. D., Saha, A., Tolstoy, E., Mateo, M., Gallagher, J.S. Hoessel, J., Chiosi, C., \& Dufour, R. J. 1997, AJ 114, 2514

Dohm-Palmer, R. C., Skillman, E. D., Gallagher, J. S., Tolstoy, E., Mateo, M., Dufour, R. J., Saha, A., Hoessel, J., \& Chiosi, C. 1998, AJ 116, 1227

Dohm-Palmer, R. C., Skillman, E. D., Mateo, M., Saha, A., Dolphin, A., Tolstoy, E., Gallagher, J. S., \& Cole, A. A. 2002, AJ 123, 813

Dolphin, A. E., et al. 2003, AJ, 126, 187

Elmegreen, B. G. 1989, ApJ, 338, 178

Elmegreen, B. G., Morris, M., \& Elmegreen, D. M. 1980, ApJ, 240, 455

Elmegreen, B. G. \& Scalo, J. 2006, ApJ, 636, 149

Engelbracht, C. W., Gordon, K. D., Rieke, G. H., Werner, M. W., Dale, D. A., \& Latter, W. B. 2005 ApJ, 628, L29

Figer, D. F. 2005, Nature, 434, 192

Gallart, C., Zoccali, M., \& Aparicio, A. 2005, ARAA, 43, 387

Garnett, D. R. 2002, ApJ, 581, 1019 
Giard, M., Bernard, J. P., Lacombe, F., Normand, P. , \& Rouan, D. 1994, A\&6A, 291, 239

Helou, G. et al. 2004, ApJS, 154, 253

Hirschi, R., Chiappini, C., Meynet, G., Maeder, A., \& Ekström, S. 2008, IAU Symposium, 250, 217

Hogg, D. W., et al. 2005, ApJ, 624, 162

Houck, J. R. et al. 2004, ApJS, 154, 211

Jackson, D. C., Cannon, J. M., Skillman, E. D., Lee, H., Gehrz, R. D., Woodward, C. E., \& Polomski, E. 2006, ApJ, 646, 192

Kennicutt, R. C., Jr. 1998, The Stellar Initial Mass Function (38th Herstmonceux Conference), 142,1

Koch, A., Grebel, E. K., Gilmore, G. F., Wyse, R. F. G., Kleyna, J. T., Harbeck, D. R., Wilkinson, M. I., \& Wyn Evans, N. 2008, AJ, 135, 1580

Leger, A. \& Puget, J. L. 1984, A\&A, 137, L5

Lemke, D., Mattila, K., Lehtinen, K., Laureijs, R. J., Liljestrom, T., Leger, A., \& Herbstmeier, U. $1998, A \mathscr{E} A, 331,742$

Lequeux, J., Le Bourlot, J., Des Forets, G. P., Roueff, E., Boulanger, F., \& Rubio, M. 1994, $A \mathscr{E} A, 292,371$

Leroy, A., Bolatto, A. D., Simon, J. D., \& Blitz, L. 2005, ApJ, 625, 763

Leroy, A., Bolatto, A., Walter, F., \& Blitz, L. 2006, ApJ, 643, 825

Leroy, A., Cannon, J., Walter, F., Bolatto, A., \& Weiss, A. 2007, ApJ, 663, 990

Lisenfeld, U. \& Ferrara, A. 1998, ApJ, 496, 145

Madden, S. C., Galliano, F., Jones, A. P., Sauvage, M. 2006, A\&AA, 446, 877

Maloney, P. \& Black, J. H. 1988, ApJ, 325, 389

Mapelli, M., Ripamonti, E., Tolstoy, E., Sigurdsson, S., Irwin, M. J., \& Battaglia, G. 2007, MNRAS, 380, 1127

Mateo, M. L. 1998, ARAA, 36, 435

Mayer, L., Governato, F., Colpi, M., Moore, B., Quinn, T., Wadsley, J., Stadel, J., \& Lake, G. 2001, ApJL, 547, L123

Mayer, L., Governato, F., Colpi, M., Moore, B., Quinn, T., Wadsley, J., Stadel, J., \& Lake, G. $2001, A p J, 559,754$

Mayer, L., Mastropietro, C., Wadsley, J., Stadel, J., \& Moore, B. 2006, MNRAS, 369, 1021

Melena, N. W., Massey, P., Morrell, N. I., \& Zangari, A. M. 2008, AJ, 135, 878

Melisse, J. P. M. \& Israel, F. P. 1994, A\&SAS, 103, 391

Miller, G. E. \& Scalo, J. M. 1979, ApJS, 41, 513

Momany, Y., Held, E. V., Saviane, I., Zaggia, S., Rizzi, L., \& Gullieuszik, M. 2007, A\&\&A, 468, 973

Norman, C. A. \& Spaans, M. 1997, ApJ, 480, 145

Oey, M. S. \& Clarke, C. J. 2005, ApJL, 620, L43

Rejkuba, M., Greggio, L., \& Zoccali, M. 2004, A\&A, 415, 915

Rosenberg, J. L., Ashby, M. L. N., Salzer, J. J., \& Huang, J.-S. 2006, ApJ, 636, 742

Röllig, M., Ossenkopf, V., Jeyakumar, S., Stutzki, J., \& Sternberg, A. 2006, A\&\&A, 451, 917

Rubio, M., Lequeux, J., \& Boulanger, F. 1993, A\&A, 271, 9

Salvadori, S., Ferrara, A., \& Schneider, R. 2008, MNRAS, 386, 348

Saviane, I., Rizzi, L., Held, E. V., Bresolin, F., \& Momany, Y. 2002, A\&A A, 390, 59

Skillman, E. D., Kennicutt, R. C., \& Hodge, P. W. 1989, ApJ, 347, 875

Skillman, E. D. \& Kennicutt, R. C., Jr. 1993, ApJ, 411, 655

Skillman, E. D., Tolstoy, E., Cole, A. A., Dolphin, A. E., Saha, A., Gallagher, J. S., DohmPalmer, R. C., \& Mateo, M. 2003, ApJ, 596, 253

Smith, J. D. T., et al. 2007, ApJ, 656, 770

Spaans, M. \& Norman, C. A. 1997, ApJ, 483, 87

Tacconi, L. J. \& Young, J. S. 1987, ApJ, 322, 681

Taylor, C. L., Kobulnicky, H. A., \& Skillman, E. D. 1998, AJ, 116, 2746

Tolstoy, E., Venn, K. A., Shetrone, M., Primas, F., Hill, V., Kaufer, A., \& Szeifert, T. 2003, AJ, 125,707

van den Bergh, S. 1994, AJ, 107, 1328 
van den Bergh, S. 1994, ApJ, 428, 617

van Dishoeck, E. F. \& Black, J. H. 1988, ApJ, 334, 771

van Zee, L., Skillman, E. D., \& Haynes, M. P. 2006, ApJ, 637, 269

Verter, F. \& Hodge, P. 1995, ApJ, 446, 616

Weidner, C. \& Kroupa, P. 2004, MNRAS, 348, 187

Weisz, D. R., Skillman, E. D., Cannon, J.M., Dolphin, A. E., Kennicutt, R. C., Jr., Lee, J., \& Walter, F. 2008, ApJ, in press.

Wilson, C. D. 1995, ApJ, 448, L97 